\title{
AN EEG SPIKE DETECTION ALGORITHM USING ARTIFICIAL NEURAL NETWORK WITH MULTI-CHANNEL CORRELATION
}

\author{
Cheng-Wen Ko*, Yue-Der Lin, Hsiao-Wen Chung, Gwo-Jen Jan \\ *Rm.238, Dept. of Electrical Engineering, National Taiwan University, \\ No.1, Section 4, Roosevelt Road, Taipei , Taiwan 10764, R.O.C. \\ *E-mail: r85165@cctwin.ee.ntu.edu.tw
}

\begin{abstract}
An automatic spike detection algorithm for classification of multi-channel electroencephalographic (EEG) signals based on artificial neural network is presented. Radial basis function (RBF) neural network was chosen for single channel recognition, with model optimization using receiver operating characteristics analysis. Waveform simplification was employed for high noise immunity. Feature extraction with as few as three parameters was used as preparation for the inputs to the neural network. Identification of multi-channel geometric correlation was performed to further lower the false-positive rate by using an incidence matrix. Threshold value for spike classification was chosen for simultaneous maximization of detection sensitivity and selectivity. Evaluation with visual analysis in this preliminary study showed a $83 \%$ sensitivity using $16-$ channel continuous EEG records of four patients, while a high false positive rate was found, which was believed to arise from the extensive and exhaustive visual analysis process. The computation time required for spike detection was significantly less than that needed for online display of the signals on the monitor. We believe that the algorithm proposed in this study is robust and that the simple structure of RBF neural network yields high potential for real-time implementation.
\end{abstract}

Keywords: spike detection, electroencephalography, radial basis function, artificial neural network, incidence matrix

\section{Introduction}

The EEG spikes are thought of as a cardinal medical sign of epilepsy [1], a detailed diagnosis of which usually demands a long-term continuous EEG monitoring. However, a long-term EEG recording generates a large amount of relatively normal but clinically useless data [2] Consequently, there has been an increasing interest in the development of computer-based automatic spike detection algorithms which would be helpful in facilitating the objective evaluation of diagnostic information.

Spike detection algorithms based on computer automation have achieved various levels of success [3-6], but there leaves ample room for improvement in terms of sensitivity, specificity, and speed. Rule-based methods [3-4] for recognizing special features of spike waveforms have achieved a high true-positive rate but as well as a high false positive rate [5]. One of the major problems leading to false spike recognition in rule-based methods lies in the fact that there is no clear definition of a spike [2]. As a result, the artificial neural network (ANN) technique for spike detection, which simulates the human reasoning process, has demonstrated strong potential since it was presented [5-6].

In this work, an automatic multi-channel spike detection algorithm based on a modified radial basis function (RBF) ANN architecture is presented. Feature extraction with as few as three parameters was implemented for simplification in training of the neural network as well as for performance efficiency. Individual classification results obtained from separate channels were sent to an incidence matrix to include geometric consideration of location of the electrical discharging sources, which effectively avoided the disadvantages of possible low specificity in single-channel recognition.

\section{Methods}

Figure 1 describes the block diagram of the entire system for multi-channel spike detection. Sixteen-channel unipolar EEG data were used with electrodes positioned according to the international $10 / 20$ system. Note that the EEG signal from each individual channel was analyzed for presence of spikes through separate classification process. Waveform simplification [7] was first applied to the original EEG signals for noise immunity. Background activity was taken into account to highlight possible spike waveform [7]. Three parameters (peak angle, amplitude and velocity) [8] were consequently extracted from the cleaned waveform and were fed to the input of the neural network for single-channel spike detection. The classification results from individual channels were then sent to an incidence matrix for identification of multi-channel geometric correlation. The results of classification were shown on the screen graphically with spikes marked out using different colors than the nonepileptic background.

The architecture of the RBF neural network for singlechannel spike detection is shown in Figure 2. The calculation of peak angle, amplitude, and velocity (i.e., slope) from the simplified EEG waveform was performed according to the definitions in Ref.[8] with voltage gain and chart speed properly adjusted. These parameters were then used as the inputs to the RBF neural network. The resulting structure thus had only five cells in the hidden layer, permitting very 


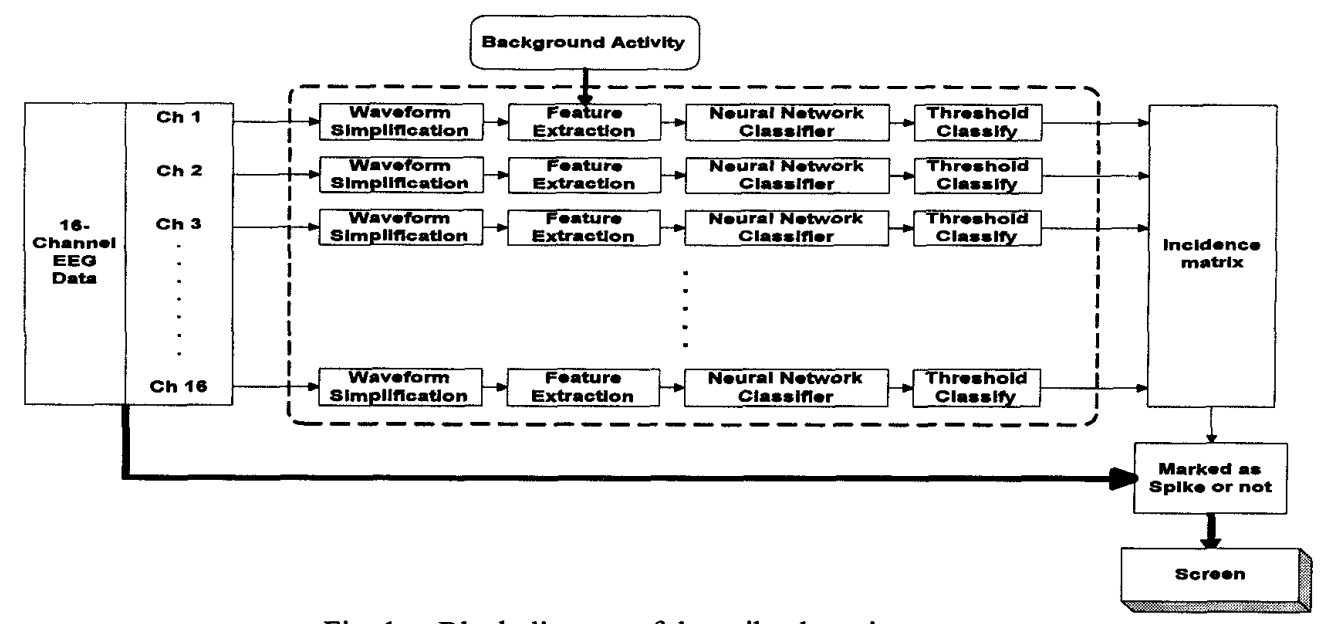

Fig. 1. Block diagram of the spike detection system.

high speed operation and relatively little training needed for optimization of the network model. Ten EEG spikes and ten nonepileptic EEG waveforms were used as the training data of the RBF neural network. In this preliminary study, three different models were evaluated, with the optimal one chosen by using receiver operating characteristics (ROC) analysis.

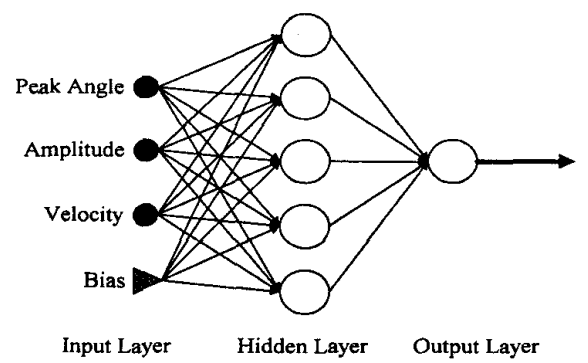

Fig. 2. The architecture of the RBF neural network with three parameters as the input.

Detection results from a single-channel RBF network contained a number ranging mostly from -1.0 to 1.0 for each data segment $330 \mathrm{msec}$ in length, representing the likelihood of being similar in features to an EEG spike. A threshold classifier was then applied for decision of the classification process. The optimal threshold value was chosen to simultaneously maximize the detection sensitivity and specificity. This was achieved by plotting the sensitivity (defined as the ratio of true positive detected by our algorithm to the total number of true spikes determined by visual analysis) and selectivity (defined as the ratio of true positive to the total number of "spikes" detected by our algorithm) as a function of varying thresholds, with the crossover point being regarded as the optimal threshold value [9]. For each time segment, the waveform with a number from the RBF network output larger than the threshold value was regarded as a spike. A " 1 " was hence assigned for such a waveform, otherwise a
"0" was assigned. The single-channel classification is thus accomplished.

Finally, the concept of the incidence matrix was employed to include identification of multi-channel geometric correlation. Assuming the epileptic spikes are from a discharging source at a specific location in the cortex, the appearance of spikes in unipolar recordings should possess geometrical correlation in that spikes tend to be grouped in neighboring channels. The identification of such a correlation was achieved by feeding the single-channel classification results as a $1 \times 16$ row vector to a $16 \times 12$ incidence matrix given below:

$\left[\begin{array}{llllllllllll}1 & 0 & 0 & 0 & 1 & 0 & 0 & 0 & 0 & 0 & 0 & 0 \\ 1 & 1 & 0 & 0 & 1 & 1 & 0 & 0 & 0 & 0 & 0 & 0 \\ 0 & 1 & 1 & 0 & 0 & 1 & 1 & 0 & 0 & 0 & 0 & 0 \\ 0 & 0 & 1 & 1 & 0 & 0 & 1 & 1 & 0 & 0 & 0 & 0 \\ 0 & 0 & 0 & 1 & 0 & 0 & 0 & 1 & 0 & 0 & 0 & 0 \\ 0 & 0 & 0 & 0 & 1 & 0 & 0 & 0 & 1 & 0 & 0 & 0 \\ 0 & 0 & 0 & 0 & 1 & 1 & 0 & 0 & 1 & 1 & 0 & 0 \\ 0 & 0 & 0 & 0 & 0 & 1 & 1 & 0 & 0 & 1 & 1 & 0 \\ 0 & 0 & 0 & 0 & 0 & 0 & 1 & 1 & 0 & 0 & 1 & 1 \\ 0 & 0 & 0 & 0 & 0 & 0 & 0 & 1 & 0 & 0 & 0 & 1 \\ 1 & 1 & 0 & 0 & 0 & 0 & 0 & 0 & 0 & 0 & 0 & 0 \\ 0 & 1 & 1 & 0 & 0 & 0 & 0 & 0 & 0 & 0 & 0 & 0 \\ 0 & 0 & 1 & 1 & 0 & 0 & 0 & 0 & 0 & 0 & 0 & 0 \\ 0 & 0 & 0 & 0 & 0 & 0 & 0 & 0 & 1 & 1 & 0 & 0 \\ 0 & 0 & 0 & 0 & 0 & 0 & 0 & 0 & 0 & 1 & 1 & 0 \\ 0 & 0 & 0 & 0 & 0 & 0 & 0 & 0 & 0 & 0 & 1 & 1\end{array}\right]$

where each column of the matrix represents each of the twelve geometrical adjacency as shown in Figure 3 . It is to be noted that each adjacency contains its neighboring channels in which spikes are expected if a discharging source is located within that certain region. Therefore, a multiplication of the $1 \times 16$ row vector obtained from the 16 RBF networks by the incidence matrix yielded a $1 \times 12$ row vector, in which each element is a number representing the likelihood of the discharging location for the spikes. Any one of the 12 elements yielding a number equal to the number 
of channels belonging to that adjacency meant that spikes were found in all channels geometrically close to the source location. A real spike was thus declared to be found. Through such an operation, false detection of eye-blinks as single-channel spikes would be eliminated, thereby reducing false-positive identification rate.

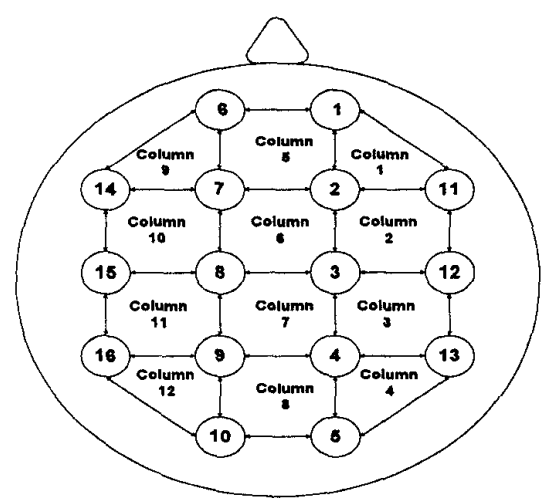

Fig. 3. The twelve adjacency used for identification of geometrical correlation. Each column in the incidence matrix represents the channels in which spikes are expected if a discharging source is located within that region.

\section{Results}

\section{On choice of neural network models}

The choice of neural network parameters were evaluated by comparing the ROC curves of three models of the RBF network. The models differed in the fact that the spikes used for training the neural network were different. In our first model, spikes with typical (within mean $+/$ - one standard deviation) peak angle, amplitude, and velocity values were selected as the training data. This has the advantage of minimizing the number of false detection, while with drawback of missing spikes with less representative feature parameters. Figure 4 shows the ROC curves plotted as detection sensitivity versus (1-specificity) varied as a function of threshold values. Model 1 was shown to exhibit high specificity but relatively low sensitivity, as expected. For comparison purpose, a second model using training data with feature parameters falling outside (mean $+/-$ std) was constructed. The ROC curve of model 2 as shown in Figure 4 demonstrated the opposite trend with low specificity, as the RBF network tended to give false identification. Model 3, obtained using training data consisting of wide spread of but centered at mean values of feature parameters, was therefore chosen. The ROC curve in Figure 4 clearly showed that model 3 had the best performance. It was thus chosen as the model for single-channel spike detection.

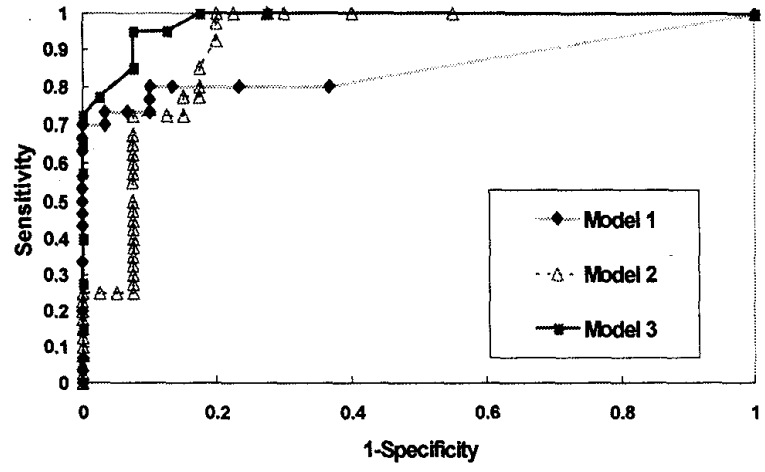

Fig. 4. ROC curves for the choice of optimal RBF neural network model.

On choice of the threshold value

Figure 5 shows the crossover curve for model 3 of the RBF network. A threshold of 0.5 was found to be the crossover point which yields high sensitivity and specificity simultaneously [9]. In our study, a threshold of 0.4 was chosen because of higher sensitivity with no loss in specificity compared with the threshold of 0.5 .

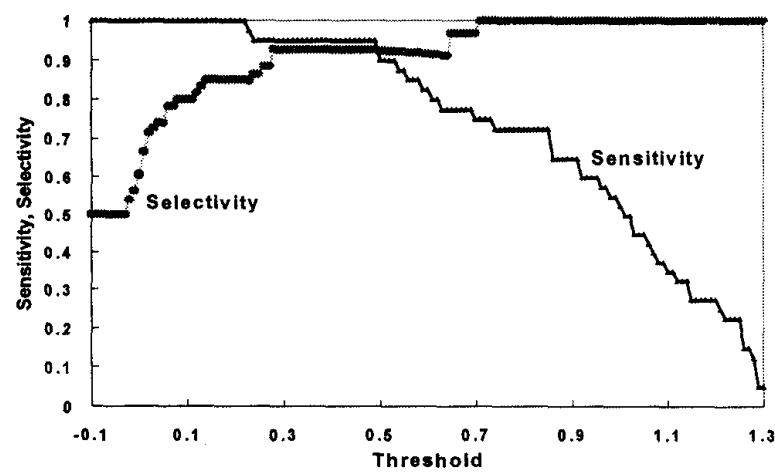

Fig. 5. Crossover curve for choosing the threshold value using model 3 of Figure 4.

\section{On the performance of the proposed algorithm}

Sixteen-channel continuous EEG records from four patients were used in the preliminary study for evaluation of spike detection algorithm. Each record was about two minutes in length. The algorithm analyzed the EEG signals in $330 \mathrm{msec}$ time segments, following which an increment of $150 \mathrm{msec}$ was added and the new time segment analyzed. On a PC system the computation time for spike detection was found to be significantly lower than that required for online display of the EEG signals. With validation by visual analysis by one experienced neural physician, 91 out of 110 spikes were detected, yielding a sensitivity of $83 \%$, although this value is subjected to statistical error due to small sample size. At the present time, however, the false positive rate was found to be unsatisfactorily high, which was believed to 
arise from the exhaustive visual analysis process. A more rigorous validation is currently underway.

\section{Discussion and Conclusions}

This study presents a new approach for the automatic detection of multi-channel EEG spikes based on RBF artificial neural network. Waveform simplification reduced the amount of the data needed to characterize spikes. Hence, together with the consideration of background activity, the RBF network was able to use as few as three feature parameters to yield the simple network structure. Consequently, a fast operation was achieved, rendering the algorithm suitable for real-time implementation.

One special feature in the proposed algorithm was the incidence matrix used for consideration of geometrical correlation among different channels. In our first experience the use of the incidence matrix significantly reduced the false identification of eye blinks or vertex waves as spikes, the results of which were unfortunately hard to be shown. In addition, the matrix multiplication was computationally inexpensive, therefore would not increase detection time even with increase in the number of EEG channels. Since the use of the incidence matrix was based on consideration of discharging source locations, it may have potential in assisting source localization.

At present, we were unable to give a complete report regarding validation of this approach, due to limited time and the exhaustive visual analysis process involved. The preliminary data suggested a sensitivity of $83 \%$, while we believed that the agreement could be higher with a more rigorous validation procedure. We believe that the algorithm proposed in this study is robust and may be helpful in facilitating objective evaluation of diagnostic information. Studies for performing a rigorous validation are currently being carried out.

\section{Acknowledgments}

The authors are indebted to Dr. Shing-Min Sung for providing the EEG data and for visual analysis validation.

\section{References}

[1] G. Bodenstein and H.M. Praetorius, "Feature extraction from the electroencephalogram by adaptive segmentation." Proc. IEEE., vol. 65, pp. 642-652, 1977.

[2] J. Gotman and J. Ives, "Computer-assisted data collection and analysis." The Treatment of Epilepsy: Principles and Practice, $2^{\text {nd }}$ ed. Elaine Wyllie, M.D. Baltimore, Williams \& Wilkins, 1996, pp. 280-291.

[3] J. Gotman and L.-Y. Wang, "State dependent spike detection: validation." Electroenceph. Clin. Neurophys., vol. 83, pp. 12-18, 1992.

[4] F. Pauri, F. Pierelli, G.E. Chatrian and W.W. Erdly, "Long-term EEG-video-audio monitoring: computer detection of focal EEG seizure patterns." Electroenceph. Clin. Neurophys., , vol. 82, pp. 1-9, 1992.
[5] W.R.S. Webber, B. Litt, K. Wilson and R.P. Lesser, "Practical detection of epileptiform discharges (EDs) in the EEG using an artificial neural network: a comparison of raw and parameterized EEG data." Electroenceph. Clin. Neurophys., , vol. 91, pp. 194-204, 1994.

[6] A.J. Gabor, R.R. Leach and F.U. Dowla, "Auotmated seizure detection using a self-organizing neural network." Electroenceph. Clin. Neurophys., vol. 99, pp. 257-266, 1996.

[7] J. Gotman, and P. Gloor, "Automatic recognition and quantification of interictal epileptic activity in the human scalp EEG." Electroenceph. Clin. Neurophys., , vol. 41, pp. 513-529, 1976.

[8] K.A. Kooi, "Voltage-time characteristics of spikes and other rapid electroencephalographic transients: semantic and morphological considerations." Neurology (Minneap.), vol. 16, pp. 59-66, 1966.

[9] W.R.S. Webber, B. Litt, R.P. Lesser, R.S. Fisher and I. Bankman, "Automatic EEG spike detection: what should the computer imitate?" Electroenceph. Clin. Neurophys., vol. 87, pp. 364-373, 1993. 